\title{
Consumo de sustancias psicoactivas en estudiantes universitarios de Ciencias de la Salud de Albacete
}

\author{
Consumption of Psychoactive Substances in University Students of Health Sciences in Albacete
}

\author{
Gloria E. Barba Fajardoํ, María Abenza Medrano², Juan Navarro Córcoles², \\ Alejandro Portero Portaz
}

\author{
'Servicio de Salud Mental del Complejo Hospitalario Universitario de Albacete. Albacete, España. \\ ${ }^{2}$ Servicio de Obstetricia y Ginecología del Complejo Hospitalario Universitario de Albacete. Albacete, España. \\ ${ }^{3}$ Servicio de Pediatría del Complejo Hospitalario Universitario de Albacete. Albacete, España. \\ Contacto: gloria.e.barba@gmail.com \\ Fecha de recepción: 12 de febrero de 2018 / Fecha de aceptación: 14 de mayo de 2018
}

\begin{abstract}
Resumen
Introducción: Hoy día, un amplio sector de la población se encuentra sometido a importantes exigencias en el ámbito académico, por la necesidad de obtener los mejores resultados posibles. Por ello, este grupo poblacional se encuentra en una situación que puede propiciar el consumo de sustancias psicoactivas con la finalidad de influir en su rendimiento académico, sin estar plenamente informados de los riesgos que puede suponer un uso inapropiado. El objetivo general es describir el patrón de consumo de diferentes sustancias utilizadas en la actualidad con la finalidad de mejorar el rendimiento académico, en la población universitaria de la rama de las Ciencias de la Salud del Campus de Albacete.

Método: Estudio observacional, descriptivo y transversal que se desarrollará en el Campus de Albacete, con la participación de los alumnos de las facultades de Medicina, Farmacia y Enfermería matriculados en el curso académico 2017-2018 y que voluntariamente acepten participar en el estudio.

Resultados: Alcohol (93,1\%), café $(86,6 \%)$ y bebidas energéticas $(84,7 \%)$ se posicionan como las sustancias psicoactivas más consumidas en la población estudiada. Si consideramos únicamente las motivaciones que influyen en el rendimiento académico, las sustancias más utilizadas son café, bebidas energéticas y té (61,2\%).

Conclusiones: La frecuencia de consumo de sustancias psicoactivas en la población universitaria estudiada es elevada, independientemente de que se utilice con fines recreativos o para influir el rendimiento académico. No obstante, se han obtenido datos que indican un consumo inferior al señalado para la población general.
\end{abstract}

Palabras clave: sustancias psicoactivas, patrón de consumo, rendimiento académico y estudiantes universitarios.

\begin{abstract}
Introduction: Today, a large segment of the population is subjected to important requirements in the academic field, by the need to achieve the best possible results. Therefore, this group of the population is in a situation that can lead to the use of psychoactive substances for the purpose of influencing their academic performance, without being fully informed of the potential risks of inappropriate use. The general objective is to describe the pattern of consumption of different substances used at present with the purpose of improving the academic performance, in the university population of the branch of the Sciences of the Health of the Campus of Albacete.

Method: Observational study, descriptive and transversal that will develop in the Campus of Albacete, with the participation of the students of the Faculties of Medicine, Pharmacy and Infirmary enrolled in the academic course 20172018 and that voluntarily accept to participate in the study.

Results: Alcohol (93,1\%), coffee (86,6\%) and drunk energetic (84,7\%) position like the psychoactive substances more consumed in the population studied. If we consider only the motivations that influence in the academic performance, the most used substances are coffee, drunk energetic and tea $(61,2 \%)$.

Conclusions: The frequency of consumption of psychoactive substances in the university population studied is elevated, independently that it use with recreational ends or to influence the academic performance. Nevertheless, they have obtained data that indicate an inferior consumption to the distinguished for the general population.
\end{abstract}

Keywords: psychoactive substances, academic performance, consumption pattern and university students. 


\section{Introducción}

El consumo de sustancias adictivas es un problema sociosanitario que está a la orden del día ${ }^{1}$. Según los resultados obtenidos en la Encuesta Domiciliaria sobre Alcohol y Drogas en España (EDADES) publicada en el año 2015, con datos pertenecientes a los años 2013 y 2014, destaca un aumento en el consumo de alcohol, tabaco e hipnosedantes en la población de 15-64 años ${ }^{2}$. No obstante, estos datos hacen referencia al consumo de diversas sustancias en la población general y no permiten obtener datos en colectivos concretos, como es la población joven universitaria. De igual manera, no permiten determinar la motivación que impulsa a su consumo ${ }^{1}$.

El comienzo de los estudios universitarios supone un gran cambio tanto a nivel personal como social, con la presencia de situaciones que repercuten en la vida de la persona y su capacidad de afrontar el estrés que esta nueva etapa produce. Si la persona cuenta con recursos suficientes para controlar estas situaciones, ello le llevará a consolidar su propia personalidad y a crear o mantener hábitos de vida saludables. Por el contrario, en caso de no contar con estas capacidades de afrontamiento, pueden aparecer problemas de tipo ansioso o depresivo, que influirán en su rendimiento académico, aumentando la vulnerabilidad ante el consumo de distintas sustancias o drogas ${ }^{3}$. En el caso de la población universitaria, se ha podido determinar en los últimos años una mayor prevalencia en el uso de estas sustancias psicoactivas, con la finalidad de mejorar el rendimiento académico ${ }^{4}$, ya sea para aumentar sus capacidades durante el estudio o para disminuir el nivel de nerviosismo generado por las exigencias académicas ${ }^{5}$. El uso de sustancias psicoactivas va en aumento en los jóvenes estudiantes, transformándose en un potencial abuso. Diferentes estudios americanos señalan una prevalencia de consumo de estimulantes del sistema nervioso central en estudiantes universitarios que alcanza hasta el $15 \%{ }^{4}$.

Los psicótropos o sustancias psicoactivas son aquellas que afectan a los procesos mentales, ejerciendo su efecto sobre el sistema nervioso central. La información recopilada en distintas investigaciones señala que la población universitaria realiza un consumo de riesgo de diferentes sustancias psicoactivas, siendo este un "patrón de consumo de sustancias que eleva el riesgo de sufrir consecuencias nocivas para el consumidor"y que puede evolucionar hacia el abuso y la adicción ${ }^{6}$.
Atendiendo a la clasificación propuesta por la Fundación de Ayuda contra la Drogadicción (FAD), las sustancias psicoactivas se pueden clasificar según actúen como estimulantes o como depresoras del sistema nervioso central ${ }^{7}$.

El consumo de diferentes sustancias es un problema de interés público cuyo estudio y análisis recae sobre los organismos públicos competentes. En España, esta función es llevada a cabo por el Ministerio de Sanidad, Servicios Sociales e Igualdad a través de la Delegación del Gobierno para el Plan Nacional sobre Drogas (DGPNSD), que realiza bienalmente la Encuesta Domiciliaria sobre Alcohol y Drogas en España (EDADES) a los residentes de 15-64 años y la Encuesta Estatal sobre el Uso de Drogas en Enseñanzas Secundarias (ESTUDES), a estudiantes de 14-18 $\operatorname{años}^{2,8}$. Ambas encuestas pasan a formar parte del Observatorio Español de Drogas y Toxicomanías (OEDT) a través de un informe global ${ }^{9}$. Posteriormente el European Monitoring Centre for Drugs and Drug Addiction (EMCDDA) realiza periódicamente un análisis completo de datos a nivel europeo ${ }^{10}$.

Sin embargo, estas encuestas poblacionales no permiten obtener datos de colectivos específicos, como es la población universitaria. Pocas de las investigaciones publicadas hasta la fecha tienen en cuenta la motivación de los entrevistados a la hora de consumir determinadas sustancias. Por ello, existen escasos estudios en los que se haya asociado este consumo con motivos estrictamente académicos. En diferentes estudios de ámbito internacional, se indagó en las razones que motivaban a los estudiantes para usar diversas sustancias, considerando otras diferentes de las recreativas. Algunas de las motivaciones obtenidas fueron: "Para prolongar el estado de vigilia", "Para aliviar el estrés", y: "Para aumentar la concentración"4,11,12.

El objetivo principal de este estudio es describir el patrón de consumo de diferentes sustancias utilizadas en la actualidad con la finalidad de mejorar el rendimiento académico, en la población universitaria de la rama de las Ciencias de la Salud del Campus de Albacete. Los objetivos secundarios son identificar el perfil sociodemográfico de los jóvenes consumidores, la prevalencia y frecuencia de consumo de las distintas sustancias, la motivación que lleva a consumirlas, el riesgo percibido por los encuestados en relación al uso de cada sustancia, identificar la posible relación entre la carrera y el curso con el consumo de sustancias, y si las horas de descanso, actividad física y el estrés perci- 
bido por los estudiantes son factores influyentes en el consumo o no de estas sustancias.

\section{Metodología}

Se llevó a cabo un estudio observacional, descriptivo y transversal realizado en el Campus Universitario de Albacete (UCLM), cuya recogida de datos tuvo lugar durante los meses de octubre, noviembre y diciembre de 2017.

\section{Participantes}

La población diana estaba constituida por 1347 alumnos pertenecientes a las facultades de Medicina, Farmacia y Enfermería de Albacete, de los cuales 426 eran estudiantes de Enfermería, 260 de Farmacia y 661 de Medicina.

Para un tamaño poblacional de 1347 (número de estudiantes matriculados en los grados de Enfermería, Farmacia y Medicina), asumiendo una prevalencia de consumo de al menos una sustancia psicoactiva del $50 \%$ (al no disponer de datos concretos en el ámbito español sobre consumo de, sustancias psicoactivas con estos fines en estudiantes universitarios), para una precisión $\pm 5 \%$ y un nivel de confianza del $95 \%$, se obtuvo que el tamaño muestral necesario era de 299 estudiantes (EpiDat 3.1).

No obstante, se encuestó de forma intencional a todos los alumnos que aceptasen participar en el estudio y que cumpliesen los criterios de inclusión determinados: ser estudiante del Campus Universitario de Albacete, formar parte de las carreras incluidas en el estudio, estar presente el día de la encuesta y aceptar la participación en el presente estudio. Fueron excluidas aquellas personas que no comprendiesen el castellano, que tuviesen capacidades cognoscitivas disminuidas o que cursasen más de una carrera.

\section{Instrumentos}

La recogida de datos se llevó a cabo mediante un cuestionario estructurado, autoadministrado, anónimo y voluntario en el que se incluyeron variables de tipo sociodemográfico, de tipo académico, datos sobre el consumo, motivos de consumo, eficacia y riesgo percibido sobre cada una, así como otros datos sobre actividad física, sueño y nivel de estrés.

\section{Análisis}

El análisis estadístico de los datos obtenidos se llevó a cabo con el programa SPSS. En el análisis descriptivo se estudiaron las frecuencias, porcentajes, medidas de tendencia central y dispersión de las distintas variables. El contraste de hipótesis se llevó a cabo con la prueba $\chi^{2}$, U Mann-Whitney o $t$ de Student para un intervalo de confianza del $95 \%$.

\section{Resultados}

\section{Datos demográficos}

La muestra analizada estuvo formada por un total de 948 estudiantes universitarios de los Grados de Enfermería, Farmacia y Medicina, de los cuales el 30,6\% eran hombres y el 69,4\% eran mujeres. La edad media de los participantes fue de 20,14 años $(\sigma=2,631)$. En relación con el estado civil, el 99,3\% eran solteros y el $0,7 \%$ restante estaba constituido por personas casadas o divorciadas. El 55,5\% de la población encuestada residía en el hogar familiar, el 15,6\% lo hacía en residencias universitarias, el $27,3 \%$ en piso compartido y el 1,3\% vivían solos. En cuanto a la descendencia, el 99,3\% de los participantes afirmó no tener hijos. Con respecto a los encuestados que compaginaban los estudios con el trabajo, se determinó que únicamente el $5,2 \%$ lo hacían, con una media de 14,85 horas de trabajo a la semana $(\sigma=10,351)$.

\begin{tabular}{|c|c|c|c|c|c|c|c|c|}
\hline & \multicolumn{6}{|c|}{ Curso } & \multirow{2}{*}{ Tota } \\
\hline & & 1 & 2 & 3 & 4 & 5 & 6 & \\
\hline \multirow{3}{*}{ Estudios } & Grado Enfermería & 94 & 87 & 62 & 30 & 0 & 0 & 273 \\
\hline & Grado Farmacia & 72 & 35 & 39 & 21 & 25 & 0 & 192 \\
\hline & Grado Medicina & 112 & 92 & 83 & 76 & 48 & 72 & 483 \\
\hline Total & & 278 & 214 & 184 & 127 & 73 & 72 & 948 \\
\hline
\end{tabular}

Tabla 1: Número de estudiantes encuestados según grado universitario y curso 
De los 948 alumnos encuestados, $273(28,8 \%)$ cursaban el Grado de Enfermería, 192 (20,3\%) el Grado de Farmacia y 483 (50,9\%) el Grado de Medicina (tabla 1). Un 4,5\% de los participantes había repetido algún curso, siendo la media de años de exceso de $1,07(\sigma=0,346)$. El 9,5\% de los alumnos señaló tener alguna asignatura pendiente, siendo la media de 2,19 asignaturas $(\sigma=1,333)$.

\section{Frecuencia de consumo}

Las sustancias psicoactivas más consumidas fueron alcohol, café y/o cápsulas y bebidas energéticas. Un 93,1\% había consumido bebidas alcohólicas alguna vez, destacando el consumo en la última semana de un $47,8 \%$. Un $86,6 \%$ de los encuestados señalaron haber consumido café y/o cápsulas, convirtiéndose en la sustancia psicoactiva de consumo diario más prevalente, con una frecuencia del $44 \%$. Resulta relevante el uso global de bebidas energéticas por parte de un $84,7 \%$ de los participantes, de los cuales el 31,5\% aseguraron haberlas consumido en la última semana previa a la encuesta. Se puede apreciar una tendencia similar en el caso del té en la población universitaria, observándose un consumo global de $61,2 \%$ con una mayor frecuencia de consumo de forma ocasional $(28,8 \%)$.

En contraposición, los estudiantes encuestados refirieron en su mayoría no haber consumido nunca anfetaminas $(98,6 \%)$, smart drugs $(98 \%)$, betabloqueantes $(97,1 \%)$ y ansiolíticos $(89 \%)$. Cabe destacar la prevalencia de consumo de estas sustancias sin prescripción médica $(84,6 \%, 100 \%, 48,1 \%$ y $43,3 \%$ respectivamente).

Los datos obtenidos indican que un porcentaje elevado de los estudiantes encuestados no consumen cannabis $(76,1 \%)$, multivitamínicos $(72,7 \%)$ ni tabaco $(73,7 \%)$, encontrándose un patrón homogéneo en cuanto a la frecuencia de consumo (tabla 2).

\begin{tabular}{|c|c|c|c|c|c|c|}
\hline Sustancias & $\begin{array}{l}\text { Nunca } \\
\text { N (\%) }\end{array}$ & $\begin{array}{c}\text { En alguna } \\
\text { ocasión } \\
\text { N (\%) }\end{array}$ & $\begin{array}{c}\text { En el } \\
\text { último mes } \\
\mathrm{N}(\%)\end{array}$ & $\begin{array}{c}\text { En la última } \\
\text { semana } \\
\text { N }(\%)\end{array}$ & $\begin{array}{c}\text { A diario } \\
\mathbf{N}(\%)\end{array}$ & $\begin{array}{c}\text { Casos } \\
\text { perdidos }\end{array}$ \\
\hline Alcohol & $65(6,9 \%)$ & $228(24,1 \%)$ & $199(21,0 \%)$ & $453(47,8 \%)$ & $2(0,2 \%)$ & 1 \\
\hline Anfetaminas & $931(98,6 \%)$ & $11(1,2 \%)$ & $1(0,1 \%)$ & $0(0,0 \%)$ & $1(0,1 \%)$ & 4 \\
\hline Ansiolíticos & $838(89,0 \%)$ & $81(8,6 \%)$ & $11(1,2 \%)$ & $6(0,6 \%)$ & $6(0,6 \%)$ & 6 \\
\hline Bebidas energéticas & $145(15,3 \%)$ & $275(29,0 \%)$ & $146(15,4 \%)$ & $298(31,5 \%)$ & $83(8,8 \%)$ & 1 \\
\hline Betabloqueantes & $913(97,1 \%)$ & $17(1,8 \%)$ & $4(0,4 \%)$ & $5(0,5 \%)$ & $1(0,1 \%)$ & 8 \\
\hline Café y/o cáps ulas & $127(13,4 \%)$ & $147(15,5 \%)$ & $55(5,8 \%)$ & $201(21,2 \%)$ & $417(44,0 \%)$ & 1 \\
\hline Cannabis & $719(76,1 \%)$ & $137(14,5 \%)$ & $53(5,6 \%)$ & $33(3,5 \%)$ & $3(0,3 \%)$ & 3 \\
\hline Multivitamínicos & $688(72,7 \%)$ & $157(16,6 \%)$ & $34(3,6 \%)$ & $23(2,4 \%)$ & $45(4,8 \%)$ & 1 \\
\hline Smart Drugs & $935(98,9 \%)$ & $6(0,6 \%)$ & $3(0,3 \%)$ & $1(0,1 \%)$ & $0(0,0 \%)$ & 3 \\
\hline Tabaco & $698(73,7 \%)$ & $88(9,3 \%)$ & $30(3,2 \%)$ & $56(5,9 \%)$ & $75(7,9 \%)$ & 1 \\
\hline Té & $367(38,8 \%)$ & $273(28,8 \%)$ & $108(11,4 \%)$ & $113(11,9 \%)$ & $86(9,1 \%)$ & 1 \\
\hline
\end{tabular}

Tabla 2: Presentación entre tipo de sustancia y frecuencia de consumo de la misma 


\section{Motivo de consumo}

El motivo de consumo de cada sustancia refleja que dentro de los consumidores de alcohol, el 89,1\% lo usa por placer y un $30,8 \%$ para socializarse. Del total de consumidores de anfetaminas, destaca que un $46,1 \%$ lo hacía por placer y un $30,7 \%$, para aumentar la concentración. Un porcentaje menor lo hace para combatir el cansancio $(23,07 \%)$ y para permanecer despierto (15,3\%). Los principales motivos de consumo de ansiolíticos son para relajarse $(61,53 \%)$ y para dormir $(39,42 \%)$, observándose un patrón de consumo similar en el caso de los betabloqueantes $(29,62 \%$ para relajarse y $18,51 \%$ para dormir). Con respecto a la motivación para consumir bebidas energéticas, se encuentra repartido entre placer $(52,4 \%)$ y para permanecer despierto $(53,1 \%)$. El café y/o las cápsulas de café es una de las sustancias psicoactivas más utilizadas en relación con el rendimiento académico, obteniéndose una importante prevalencia de personas que lo utilizan para permanecer despierto $(71,82 \%)$ y para combatir el cansancio $(71,82 \%)$. El consumo de cannabis está asociado a motivos recreativos en un $86,3 \%$ ). Los multivitamínicos son consumidos sin consejo médico por un $59,4 \%$ de la población estudiada. Entre los que hacen uso de estas sustancias, un 35,5\% lo consume para combatir el cansancio y un $26,2 \%$ para aumentar la concentración. Lo más característico del consumo de smart drugs es que, dentro de los consumidores, el $50 \%$ refiere consumirlas para aumentar su concentración y que todos las adquieren sin prescripción facultativa. Los datos obtenidos sobre el uso del tabaco y del té reflejan que el principal motivo de su consumo es por placer $(78,3 \%$ y $76,6 \%$ respectivamente $)$.

\section{Eficacia del consumo}

En cuanto al análisis de la eficacia, se observa que todas las sustancias en su mayoría fueron catalogadas como eficaces. Destacan el alcohol $(93,9 \%)$, el café $(93,7 \%)$ y los betabloqueantes $(92,0 \%)$ como las mejor valoradas en cuanto a eficacia. En sentido opuesto, llama la atención que sustancias como el tabaco, cuyo motivo más prevalente es el placer, un $23,1 \%$ de los encuestados lo considera ineficaz. Los multivitamínicos son la segunda sustancia peor valorada en cuanto a eficacia con un $20,5 \%$ (gráfico 1 ).

\section{Riesgo percibido}

En referencia al riesgo percibido para cada sustancia por los estudiantes encuestados, un $87,8 \%$ considera

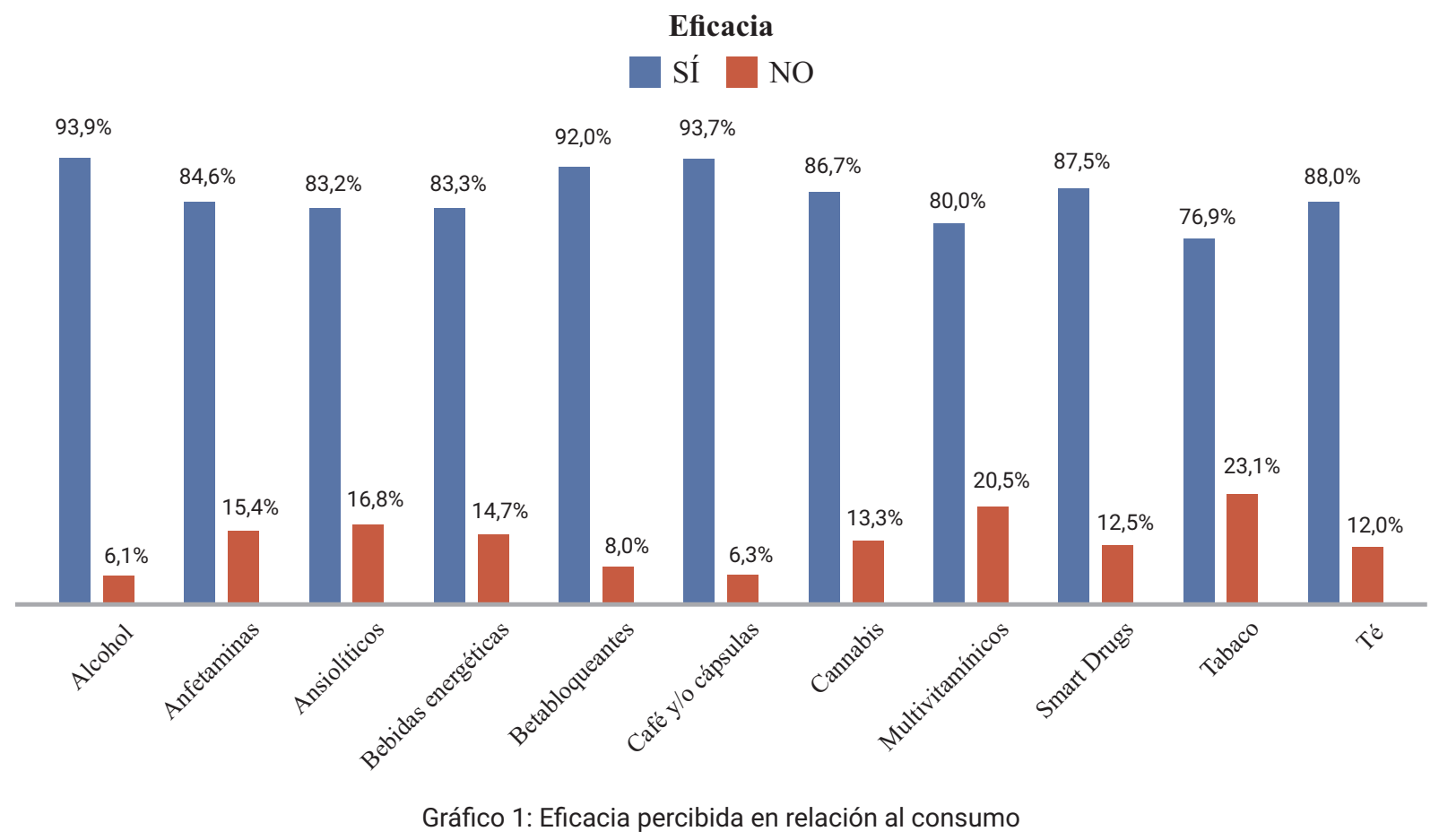


que el consumo de anfetaminas conlleva un alto riesgo para la salud, seguido del tabaco con un $74,9 \%$. Sustancias como el alcohol y los ansiolíticos han sido valoradas con un riesgo medio para la salud en un $59,1 \%$ y $48,3 \%$ respectivamente. En algunas sustancias, la valoración del riesgo se encuentra dividida en dos categorías, como es el caso de las bebidas energéticas, cuyo riesgo asignado por la población universitaria fue de medio $(45,1 \%)$ y bajo $(41,2 \%)$, o del té con un riesgo bajo $(41,9 \%)$ o ninguno $(41,4 \%)$. En cuanto a los betabloqueantes, existe discrepancia en cuanto al riesgo percibido, repartiéndose en un $32,3 \%$ al riesgo alto, $30,4 \%$ al riesgo medio y un $26,8 \%$ de la muestra que afirma desconocer el riesgo de esta sustancia. Con la asignación de riesgo bajo, destaca el café con un $58,7 \%$ y los multivitamínicos con un $40,1 \%$. Un importante sector de la población encuestada reconoce desconocer los riesgos para la salud secundarios del consumo de betabloqueantes $(26,8 \%)$, smart drugs $(19,5 \%)$, multivitamínicos $(14,7 \%)$ y ansiolíticos $(8,5 \%)$ (tabla 3$)$.

\section{Otros datos}

En cuanto a las horas de sueño, encontramos que el $70,7 \%$ de los estudiantes encuestados refiere dormir entre 5 y 7 horas, seguido del $28,4 \%$ que manifiesta dormir de 8 a 10 horas.

Con respecto a la práctica de ejercicio físico, se observa una clara variabilidad en las respuestas. El $36,6 \%$ de los estudiantes realiza ejercicio de dos a tres veces a la semana, seguido del $29,6 \%$ que lo practica menos de una vez a la semana, el 18,8\% una vez a la semana, y el $15 \%$ cuatro o más veces a la semana.

Al analizar los datos obtenidos sobre el nivel de estrés subjetivo de los alumnos encuestados, el 44,8\% de estos manifiesta un nivel de estrés medio. Destacando que sólo un $6,1 \%$ de la muestra refiere un nivel de estrés muy alto. Este dato se mantiene estable independientemente de que la muestra sea analizada en su conjunto o se haga teniendo en cuenta los estudios cursados.

\begin{tabular}{|c|c|c|c|c|c|c|}
\hline Sustancias & $\mathbf{N}$ & Alto & Medio & Bajo & Ninguno & No sabe \\
\hline Alcohol & 946 & $32,7 \%$ & $59,1 \%$ & $7,9 \%$ & $0,2 \%$ & $0,1 \%$ \\
\hline Anfetaminas & 938 & $\mathbf{8 7 , 8 \%}$ & $8,0 \%$ & $0,5 \%$ & $0,1 \%$ & $3,5 \%$ \\
\hline Ansiolíticos & 936 & $32,4 \%$ & $\mathbf{4 8 , 3 \%}$ & $9,9 \%$ & $0,9 \%$ & $\mathbf{8 , 5 \%}$ \\
\hline Bebidas energéticas & 946 & $9,7 \%$ & $\mathbf{4 5 , 1} \%$ & $41,2 \%$ & $3,0 \%$ & $1,0 \%$ \\
\hline Betabloqueantes & 929 & $\mathbf{3 2 , 3} \%$ & $30,4 \%$ & $9,4 \%$ & $1,2 \%$ & $\mathbf{2 6 , 8} \%$ \\
\hline Café y/o cápsulas & 941 & $2,1 \%$ & $23,3 \%$ & $\mathbf{5 8 , 7 \%}$ & $14,9 \%$ & $1,1 \%$ \\
\hline Cannabis & 932 & $50,0 \%$ & $34,1 \%$ & $12,9 \%$ & $1,2 \%$ & $1,8 \%$ \\
\hline Multivitamínicos & 938 & $4,2 \%$ & $13,3 \%$ & $\mathbf{4 0 , 1 \%}$ & $27,7 \%$ & $\mathbf{1 4 , 7 \%}$ \\
\hline Smart Drugs & 928 & $\mathbf{5 6 , 9 \%}$ & $18,0 \%$ & $4,5 \%$ & $1,1 \%$ & $\mathbf{1 9 , 5 \%}$ \\
\hline Tabaco & 935 & $\mathbf{7 4 , 9 \%}$ & $20,9 \%$ & $2,8 \%$ & $0,5 \%$ & $1,0 \%$ \\
\hline Té & 938 & $1,1 \%$ & $8,1 \%$ & $\mathbf{4 1 , 9 \%}$ & $\mathbf{4 1 , 4 \%}$ & $7,6 \%$ \\
\hline
\end{tabular}

Tabla 3: Riesgo percibido para cada una de las sustancias 


\section{Contraste de hipótesis}

En relación al patrón de consumo según género, se encontraron diferencias significativas en cuanto al té, las bebidas energéticas y el cannabis. Las mujeres se posicionaron como mayores consumidoras de té, de forma estadísticamente significativa $(p=0,0001)$. Ocurre lo contrario con el consumo de bebidas energéticas y cannabis, siendo ambas más consumidas por los hombres, con nivel de significación $\mathrm{p}=0,042 \mathrm{y}$ $\mathrm{p}=0,0001$ respectivamente.

No se encontraron diferencias significativas en cuanto al consumo de sustancias psicoactivas y el lugar de convivencia de los alumnos encuestados, con excepción del alcohol, para el cual se observó que aquellos que compartían piso consumían ligeramente más alcohol que otros que vivían en el hogar familiar o en residencia universitaria $(p=0,041)$.

Al analizar los datos de forma independiente, se observó relación estadísticamente significativa entre el consumo de sustancias psicoactivas y el curso estudiado, sin que suponga un patrón estable de forma global. Los alumnos que cursaban cuarto curso consumían alcohol en mayor frecuencia $(p=0,020)$, mientras que los alumnos de sexto eran mayores de consumidores de ansiolíticos $(p=0,0001)$, cannabis $(p=0,0001)$ y té $(p=0,0001)$. Los alumnos de tercer curso consumían con mayor frecuencia multivitamínicos $(p=0,0001)$. El consumo de bebidas energéticas se señaló de forma equitativa a lo largo de los distintos cursos, con un menor consumo por parte de los estudiantes de quinto curso.

El consumo de bebidas energéticas, café, cannabis y té fue superior en los estudiantes de grado de Medicina, apreciándose asociación estadísticamente significativa. Los alumnos con un nivel de estrés subjetivo alto o muy alto fueron mayores consumidores de multivitamínicos y té $(\mathrm{p}=0,0001$ y $\mathrm{p}=0,013)$.

\section{Discusión y conclusiones}

En el presente estudio, se indagó sobre el uso de sustancias psicoactivas, siguiendo la tendencia iniciada por otras investigaciones. Se consideraron como motivaciones de consumo las recreativas y las relacionadas con el rendimiento académico ${ }^{1,5,14,15,17}$.

Según los datos arrojados por la encuesta EDADES, las sustancias más consumidas por la población gene- ral son alcohol (78,3\%), tabaco (40,7\%) e hipnosedantes $(12,2 \%)^{2}$. Estos datos no difieren en exceso de los obtenidos en el presente estudio, considerando que sustancias con una alta prevalencia de consumo como son el café y las bebidas energéticas no son tenidas en cuenta en la citada encuesta.

Se encontraron coincidencias en cuanto a las elevadas tasas de consumo de alcohol, posicionándose como la sustancia psicoactiva más consumida por la población universitaria $(93,1 \%)$, con datos superiores a los determinados por la encuesta poblacional $(78,3 \%)^{2}$. Por tanto, se aprecia una tendencia creciente en el consumo de alcohol en la población universitaria encuestada con respecto a la población general, propiciada por la alta tasa de consumo de alcohol en la última semana (47,8\%). Dicha encuesta arroja datos sobre el consumo de alcohol en forma de "atracón", que resultaría interesante indagar en nuestro medio en futuras investigaciones. Igualmente resultaría de interés determinar casos de consumo de riesgo y de posible dependencia al alcohol mediante el test AUDIT. Con respecto al género, aunque no se encontró relación significativa, los resultados muestran que las mujeres consumen alcohol con mayor frecuencia que los hombres, al contrario de lo determinado en gran parte de las investigaciones previas ${ }^{1,2,4,12}$.

El consumo de tabaco ha experimentado una tendencia decreciente en los últimos años, propiciado por en gran medida por la conocida ley antitabaco. Aunque en la encuesta EDADES la prevalencia de consumo de tabaco se sitúa en un 40,7 \% en el último mes y un consumo diario del $30,8 \%{ }^{2}$, estos datos no son concordantes con nuestro estudio, en el cual se ha observado una prevalencia del consumo del $26,3 \%$, con un consumo diario del 7,9\%. Podemos afirmar por tanto que los estudiantes universitarios de la rama de las ciencias de la salud de Albacete consumen tabaco en mucha menor proporción que la población general. En estudios previos, se observó un patrón de consumo superior entre mujeres. Este cambio social apreciado en las estudiantes universitarias no queda reflejado en nuestro estudio $^{11}$. Aunque el tabaco es una droga estimulante, en la bibliografía consultada, se observó que la mayoría de los fumadores considera que actúa como un relajante ${ }^{7}$. El 23,1\% de los consumidores de tabaco de nuestro estudio clasificó su uso como ineficaz. Esto puede deberse a que un $27,7 \%$ lo consume para relajarse siendo el tabaco una sustancia estimulante. 
Los derivados del cannabis constituyen la sustancia ilegal más consumida en nuestro entorno ${ }^{7}$. La encuesta EDADES señala una prevalencia de consumo del $6,6 \%$, observándose una tendencia descendente en la última década ${ }^{2}$. Por el contrario, en el presente estudio encontramos una prevalencia del $23,9 \%$ siendo mayor significativamente el consumo en hombres que en mujeres $(32,1 \%$ frente a $20,3 \%, p=0,0001)$.

Los resultados obtenidos sobre policonsumo de sustancias utilizadas para mejorar el rendimiento académico en los estudiantes universitarios de la rama de las ciencias de la salud de Albacete evidencian que un $87,4 \%$ de los participantes consumen más de una sustancia para mejorar el rendimiento académico. Estos datos contrastan con los aportados por Asencio et al., quienes afirmaban que un $92,5 \%$ de los estudiantes de la encuesta de Medicina de la Universidad de Valparaíso consumía al menos una sustancia para mejorar el rendimiento académico (café, multivitamínicos, modafinilo, etc.), de los cuales el 14,2\% empleaba sustancias ilícitas (anfetaminas, betabloqueantes, marihuana, etc.) con similar objetivo. Otros estudios realizados en estudiantes universitarios muestran una prevalencia sobre policonsumo inferior a la obtenida en el presente estudio, encontrándose en torno a un $52 \%$ en el caso de la universidad de Medicina de Manizares ${ }^{5,14}$.

La sustancia usada con mayor frecuencia con intención de influir en el rendimiento fue el café $(86,6 \%)$, seguido de las bebidas energéticas $(84,7 \%)$, el té $(61,2 \%)$ y los multivitamínicos $(27,3 \%)$. En la actualidad, la cafeína se posiciona como la sustancia psicoactiva más ampliamente consumida en el mundo, obteniéndose prevalencias similares a las del presente estudio $^{5,13-15}$. Según el estudio publicado por la European Food Safety Authority (EFSA) en el que se analiza el consumo de bebidas energéticas a nivel europeo, se ha visto que los adolescentes (10-14 años) son el grupo de edad con mayor riesgo de consumo de estas sustancias $(68 \%)^{16}$. En el presente estudio, se observa una frecuencia de consumo en estudiantes universitarios del $84,7 \%$. Esto nos lleva a reflexionar sobre la necesidad de realizar educación para la salud en etapas tempranas para prevenir el creciente uso de estas sustancias. El consumo en la última semana de las bebidas energéticas es mayor en estudiantes universitarios $(31,5 \%)$ que en los encontrados sobre la población general $(12 \%)^{16}$, con una importante asociación a la motivación "para permanecer despierto", por lo que podemos asumir que estas sustancias son consumidas en gran medida para influir en el rendimiento académi- co. Al relacionar el sexo con el uso de sustancias estimulantes, sólo se encontraron diferencias significativas en cuanto al té. Observándose que el sexo femenino hace uso de esta sustancia con mayor frecuencia que el sexo masculino $(p=0,0001)$. En el resto de sustancias no se encontró relación significativa al igual que en otros estudios ${ }^{15}$.

Resultan alarmantes los datos obtenidos en relación con la adquisición mediante prescripción facultativa o no de sustancias sometidas a la misma. Se ha podido determinar que anfetaminas, ansiolíticos, betabloqueantes y smart drugs fueron consumidas sin prescripción médica en un $84,6 \%, 43,3 \%, 48,1 \%$ y $100 \%$ de los casos. Sería interesante en posteriores estudios indagar sobre la procedencia y los medios de acceso a dichas sustancias como se ha llevado a cabo en otros estudios $^{17}$. De forma similar, los multivitamínicos fueron consumidos por recomendación facultativa en un $40,5 \%$ de los casos, dato que supera en gran medida al obtenido en una investigación previa llevada a cabo en Colombia $(14,8 \%)^{18}$.

De la misma manera, resulta llamativo que la población encuestada refiera desconocimiento sobre el riesgo secundario al consumo de betabloqueantes (26,8\%), smart drugs (19,5\%), multivitamínicos $(14,7 \%)$ y ansiolíticos $(8,5 \%)$. Estos datos resultan todavía más llamativos si tenemos en cuenta que se trata de población estudiante con formación en farmacología.

En conclusión, en la muestra de alumnos encuestados, se registró una alta prevalencia de consumo de sustancias psicoactivas, tanto con fines recreativos como con fines relacionados con el rendimiento académico. Aunque la prevalencia de consumo para mucha de las sustancias es inferior a la obtenida en población general, sería beneficioso seguir analizando datos que no han sido tenidos en cuenta en el presente estudio, así como implementar herramientas pedagógicas centradas en aumentar la información sobre los efectos y riesgos asociados al consumo de las sustancias psicoactivas. 


\section{Bibliografía}

1. Martín-Montañez E, Barón-López FJ, Lamia LR, Molina JP, Páez JM, Amaya IS. Consumo de alcohol, tabaco, cannabis y otras sustancias psicoactivas en estudiantes de la Universidad de Málaga. Trastor adict. 2011;13(4): 160-166.

2. Delegación del Gobierno para el Plan Nacional Sobre Drogas. Informe de la encuesta domiciliaria sobre alcohol y drogas de España (EDADES). Madrid: Delegación del Gobierno para el Plan Nacional Sobre Drogas; 2015 . Disponible en: http://www.pnsd.msssi.gob.es/profesionales/sistemasInforma cion/sistemalnformacion/pdf/2015_Informe_EDADES.pdf

3. Fernández T, Alguacil J, Ayán C, Bueno Aurora, Candela JM, Capelo R et al. Proyecto UNIHCOS: cohorte dinámica de estudiantes universitarios para el estudio del consumo de drogas y otras adicciones. Rev. Esp. Salud Pública [revista en Internet] 2013 diciembre [citado 21 de diciembre de 2015]; 87(6): 575-585. Disponible en: http://scielo.isciii.es/scielo.php?pid=S1135$57272013000600003 \&$ script=sci_arttext

4. Gloria-Garcés C, Graziani K. Consumo de estimulantes del sistema nervioso central en estudiantes de enfermería y medicina de una universidad chilena. SMAD revista electrónica salud mental, alcohol y drogas [revista en Internet] 2013 mayo-agosto [citado 26 de octubre de 2015]; 9(2): 64-69. Disponible en: http://pepsic.bvsalud.org/scielo.php?pid=S180669762013000200003\&script=sci_arttext\&tlng=es

5. Asencio F, Cardemil F, Betancour P. Prevalencia y factores asociados al uso de drogas, fármacos y otras sustancias por estudiantes de Medicina para aumentar el rendimiento. CIMEL. 2009; 14(2): 8792.

6. World Health Organization. Glosario de términos de alcohol y drogas; 1994.

7. Fad.es [citado 26 de octubre de 2015]. Disponible en: www.fad.es/sustancias.

8. Delegación del Gobierno para el Plan Nacional Sobre Drogas. Informe de la encuesta estatal sobre el uso de drogas en estudiantes de secundarias (ESTUDES). Madrid: Delegación del Gobierno para el Plan Nacional sobre Drogas; 2012. Disponible en: http://www.pnsd.msssi.gob.es/profesionales/sistemasInforma cion/sistemalnformacion/pdf/8_ESTUDES_2012_Informe.pdf

9. Observatorio Europeo de las Drogas y las Toxicomanías. Informe anual 2010: el problema de la drogodependencia en Europa. Luxemburgo: Oficina de publicaciones de la Unión Europea. 2010; 106-107. Disponible en:

http://www.emcdda.europa.eu/attachements.cfm/att_120104_ ES_EMCDDA_AR2010_ES.pdf

10. Observatorio Europeo de las Drogas y las Toxicomanías. Informe Europeo sobre Drogas. Luxemburgo: Oficina de publicaciones de la Unión Europea; 2015. Disponible en:

http://www.emcdda.europa.eu/attachements.cfm/att_239505_ ES_TDAT15001ESN.pdf

11. Jiménez-Muro A, Beamonte A, Maqueta A, Gargallo P, Nerín I. Consumo de drogas en estudiantes universitarios de primer curso. Adicciones. 2009; 21(1): 21-28.

12. Viña CM, Herrero M. El consumo de sustancias psicoactivas en estudiantes de Psicología de la Universidad de La Laguna. IJCHP. 2004; 4(3): 521-536.

13. Osada JE, Rojas MO, Rosales CE, Vega J. Consumo de cafeína en estudiantes de medicina y su coexistencia con sintomatología ansiosa y depresiva. Rev Med Hered. 2008; 19(3): 102-107.

14. Barón L, Botero K, Castaño JJ, Castillo K, Díaz J, Echeverri JS et al. Prevalencia y factores asociados al consumo de anfetaminas, en estudiantes del programa de medicina de la universidad de Manizales. Rev Fac Med. 2011; 59: 201-204.

15. Buahanan J, Pillon S. Uso de drogas entre estudiantes de medicina, Tegucigalpa, Honduras. Rev Latino-am Enfermagem. 2008; 16: 595-600.
16. Agencia Española de Consumo, Seguridad Alimentaria y Nutrición [sede Web]. Madrid: Ministerio de Sanidad, Servicios Sociales e Igualdad. Informe sobre bebidas "energéticas". Disponible en: http://www.aecosan.msssi.gob.es/AECOSAN/web/home/aeco san_inicio.shtml

17. Mazzoglio MJ, Algieri RD, Dogliotti CB, Gazzotti AM, Jiménez HN, Rey LM. Utilización de sustancias psicoactivas en alumnos de anatomía y su implicación en el aprendizaje. Educ Med. 2011; 14(2): 129-132.

18. Martínez GI, Martínez LM, Rodríguez MA, Jiménez JG, Lopera JS, Vargas $\mathrm{N}$ et al. Prevalencia y características del consumo de nutracéuticos en estudiantes universitarios de grado. Investigaciones Andina. 2013;17(31): 1341-1353. 